\title{
Elevated levels of neutrophil gelatinase- associated lipocalin among OCD patients: an exploratory study
}

\author{
Catarina Raposo-Lima ${ }^{1,2}$, Inês Miguel Pereira ${ }^{1,2}$, Fernanda Marques ${ }^{1,2+}$ and Pedro Morgado ${ }^{1,2,3^{*}+}$
}

\begin{abstract}
Background: Obsessive-compulsive disorder (OCD) is a debilitating psychiatric disease that is characterized by its clinical heterogeneity and complex pathophysiology. This complexity comes from the diversity of pathophysiological factors that have been proposed to be involved in the natural history of the disorder. Many theories on OCD pathology support inflammation as a pathophysiological factor, although studies are not consistent on the presence of a proinflammatory state among OCD patients. However, some pre-clinical animal studies suggest lipocalin-2 (LCN2), an analogous form of the acute-phase pro-inflammatory protein neutrophil gelatinase-associated lipocalin (NGAL), may be involved in in the regulation of the stress response, which is thought to be disrupted in OCD.

Methods: Twenty-one OCD patients and 19 healthy subjects participated in this exploratory study. Levels of NGAL were assessed in the peripherous blood of all participants. Severity of disease was assessed using the Yale-Brown ObsessiveCompulsive Scale (Y-BOCS).
\end{abstract}

Results: OCD patients exhibited significantly higher levels of NGAL when compared to healthy control subjects. No correlation was found between elevated levels of NGAL and severity of symptoms.

Conclusions: This is the first study to report elevated levels of NGAL among OCD patients, adding evidence for a possible role of immune dysregulation in the pathophysiology of OCD.

Keywords: Obsessive-compulsive disorder, Neutrophil gelatinase-associated lipocalin, Lipocalin, Immunology, Immune dysregulation

\section{Background}

Obsessive-compulsive disorder (OCD) is a widely prevalent psychiatric disease that is characterized by the presence of one or two core symptoms, obsessions and compulsions [1]. This chronic and debilitating disease is as heterogenous in presentation as it is in nature, with multiple factors proposed to influence its pathophysiology, from genes to environmental factors, encompassing infection, vitamin

\footnotetext{
* Correspondence: pedromorgado@med.uminho.pt

${ }^{\dagger}$ Fernanda Marques and Pedro Morgado contributed equally to this work. 'Life and Health Sciences Research Institute (ICVS), School of Medicine, University of Minho, 4710-057 Braga, Portugal

${ }^{2}$ ICVS-3Bs PT Government Associate Laboratory, Braga, Guimarães, Portugal Full list of author information is available at the end of the article
}

deficiencies, stressful life events, brain injury and personality, to cite a few [2]. Since the first report by Swedo et all [3] of a form of OCD arising after streptococcal infection, PANDAS (pediatric autoimmune neuropsychiatric disorder associated with streptococcal infections), many authors have studied the role of inflammation in OCD patients, hypothesizing a role for immune dysregulation in OCD pathophysiology.

In PANDAS, obsessive-compulsive symptoms (OCS) occur in the context of group A $ß$-hemolytic streptococcal (GABHS) infection, in which anti-streptococcal antibodies are believed to cross-react with epitopes expressed in neuronal cells of the basal ganglia (particularly the caudate

(c) The Author(s). 2021 Open Access This article is licensed under a Creative Commons Attribution 4.0 International License, which permits use, sharing, adaptation, distribution and reproduction in any medium or format, as long as you give appropriate credit to the original author(s) and the source, provide a link to the Creative Commons licence, and indicate if changes were made. The images or other third party material in this article are included in the article's Creative Commons licence, unless indicated otherwise in a credit line to the material. If material is not included in the article's Creative Commons licence and your intended use is not permitted by statutory regulation or exceeds the permitted use, you will need to obtain permission directly from the copyright holder. To view a copy of this licence, visit http://creativecommons.org/licenses/by/4.0/ The Creative Commons Public Domain Dedication waiver (http://creativecommons.org/publicdomain/zero/1.0/) applies to the data made available in this article, unless otherwise stated in a credit line to the data. 
and putamen), a key region involved in OCD expression [4-6]. Adding to this hypothesis are studies using GABHS exposure in animal models, which has shown to result in multiple locomotor and cognitive deficits, including anxiety and stereotyped behavior, and increased IgG antibodies and receptors within the striatum, thalamus and frontal cortex, all regions known to be involved in the pathophysiology of OCS (for further details on this subject see the work by Gerentes and colleagues [7] and Mora and colleagues [8]). Although PANDAS might be of relevance for only a minor proportion of OCD cases, this immune/inflammation hypothesis may be significant given the prevalence of OCS among patients with autoimmune diseases [9].

In that regard, many reports on immunology and OCD suggest an increase in proinflammatory markers among OCD patients [7, 10-12], including within the cortico-striatal-thalamo-cortical (CSTC) circuit [13], although recent systematic reviews and meta-analysis point in a different direction, with no differences between OCD patients and controls in what concerns levels of inflammatory cytokines and C-reactive protein $[14,15]$. This disparity is due to major methodological differences across studies, with sample sizes, matching of individuals, age of participants, presence of comorbidities and medication status as key limiting factors $[10,14$, 15]. Notwithstanding the conflicting literature on the subject, the role of immune dysregulation in OCD should not be overlooked since it might be an important factor in a particular subset of patients, where autoimmunity might be triggered by specific pathogenic agents with similar surface epitopes in the central nervous system (CNS), which may be the case, for instance but not exclusively, of patients with prior history of GABHS infection [16]. Additionally, it is possible that a combination of etiological factors, with specific mechanisms yet incompletely understood, is involved in the development of OCD, and thus the possible involvement of inflammation cannot be ruled out [2].

On this regard, in spite of the inconsistencies across studies and following the hypothesis for the presence of a pro-inflammatory state among OCD patients, we conducted an exploratory study with a small subset of patients by evaluating the levels of plasma neutrophil gelatinase-associated lipocalin (NGAL). NGAL is an acute-phase pro-inflammatory protein [17] that is involved in iron homeostasis [18] and in spine morphology and neuronal excitability in the CNS [19]. Increased levels of NGAL have been detected among multiple sclerosis (MS) patients [20] as well as in heart failure, stroke and coronary artery disease, where it has prognostic significance [21]. It has also been associated with depressive symptom severity $[22,23]$, although this later finding remains to be proven [24]. In rodents, lipocalin-
2 (LCN2) - the analogous form of NGAL - has been shown to be expressed in response to peripheral inflammation [25], focal brain ischemia [26] and spinal cord injury $[27,28]$. It is also up-regulated during the active phase of an animal model for MS [20] and seems to be involved in the pathophysiology of Alzheimer's disease $[29,30]$ and Parkinson's disease [31].

Most interestingly, a study using LCN2-null mice showed these animals exhibit anxiety- and depressivelike behavior and cognitive impairments in spatial learning tasks that correlated with increased hypothalamicpituitary-adrenal (HPA) axis activity and morphological alterations within the hippocampus, specifically, an atrophy of the dorsal hippocampus - which is involved in memory and cognition - and a hypertrophy of the ventral region - which plays a role in emotion. Furthermore, neuronal morphology and dendritic spine density within the hippocampus were altered in mutant mice [32]. This is in accordance with previous studies suggesting a role for LCN2 in the regulation of neuronal morphology and excitability in both the hippocampus and basolateral amygdala in response to stress [33, 34]. Further work with LCN2-null mice demonstrated LCN2 is involved in adult neurogenesis, differentiation and survival, where impairments at this level translate into poor performance in a hippocampal-dependent contextual fear discriminative task [35].

It is noteworthy, however, that both the morphological and behavioral changes observed in LCN-2 null mice are in line with those observed in animals after chronic stress exposure or prolonged administration of exogenous glucocorticoids [36], which raises the question whether or not LCN2 is also involved in the regulation of the stress response, a feature that is known to be disrupted in a number of psychiatric diseases, for instance, OCD.

\section{Methods \\ Subjects}

A sample of 40 subjects (21 OCD patients, 19 controls) without previous history of neurological disorders participated in this study. OCD patients were recruited from the Psychiatric outpatient clinic of Hospital de Braga, Portugal, over a period of 3 months, and characterized with a comprehensive clinical assessment. The diagnosis of the disorder was established by experienced psychiatrists, using a semi-structured interview based on Diagnostic and Statistical Manual of Mental Disorders, Fifth Edition (DSM-5). Severity of disease was assessed using the Yale-Brown Obsessive-Compulsive Scale (Y-BOCS) $[37,38]$. This clinical assessment allowed for the exclusion of presence of other psychiatric diagnoses, particularly depression and other anxiety disorders. Because one female OCD patient was in clinical remission and 
asymptomatic at the time of the analysis, this subject was excluded from the study.

All patients were under current pharmacological treatment, primarily a selective serotonin reuptake inhibitor (fluvoxamine, sertraline or fluoxetine).

Healthy controls were recruited to match OCD patients for age and gender.

\section{Blood sampling and analysis}

Serum samples were obtained according to standardized protocols in the Hospital de Braga, Portugal, at the time of recruitment. Human Lipocalin-2/NGAL Quantikine ${ }^{\circ}$ ELISA Kit (R\&D Systems, Inc., Minneapolis, USA) was used according to the manufacturer's instructions. Briefly, a 96-plate was incubated with $100 \mu \mathrm{L} /$ per well of Assay Diluent RD1-52, and $50 \mu \mathrm{L}$ of sample was added to each well. The plate was covered and incubated for 2 $\mathrm{h}$, at $8{ }^{\circ} \mathrm{C}$. Then, it was washed four times with Wash Buffer $(400 \mu \mathrm{L})$, and $200 \mu \mathrm{L}$ of Human Lipocalin-2 Conjugate was added to each well. After an incubation step of $2 \mathrm{~h}\left(8^{\circ} \mathrm{C}\right)$, the plate was washed four times. At the end, $200 \mu \mathrm{L}$ of Substrate Solution was added and incubated for $30 \mathrm{~min}$ at room temperature, with the plate covered to protect it from light. Finally, after adding $50 \mu \mathrm{L}$ of the Stop Solution, all samples were quantified by determining optical density in a spectrophotometer set to $450 \mathrm{~nm}$ or $450(-570) \mathrm{nm}$.

\section{Data processing and analysis}

Statistical analysis for sociodemographic and clinical data was performed using IBM SPSS 27 software [39]. All hypothesis tests had a level of significance $(p)$ set at 0.05 . Shapiro-Wilk was used to test variables for normal distribution. Since normality was not met, mean comparisons were performed using Mann-Whitney test. Correlations were performed using Spearman's rank correlation.

\section{Ethics statement}

This study was performed in accordance with the Declaration of Helsinki and was approved by Ethics Subcommittee for the Life and Health Sciences of University of Minho, Portugal, and by the Ethics Committee of the Hospital of Braga, Portugal. All subjects were provided with written informed consent following description of the study goals and procedures.

\section{Results}

\section{Socio-demographic and clinical characteristics}

The groups were similar in respect to age and gender [control group (CONT), $n=19 ; 10$ females/9 males; median and interquartile range (IQR) 28.00 (5) years of age; OCD group, $n=20$; 9 females/11 males; 30.00 (7) years of age] (see Table 1 for further details).
Total Y-BOCS score ranged from 9 to $38(\mathrm{n}=20$, mean \pm standard deviation (SD) $26.10 \pm 1.56$, with scores for compulsions and obsession subscales ranging from 4 to 18 and 5 to 20, respectively (compulsions mean score $11.50 \pm 0.76 \mathrm{SD}$; obsessions mean score $14.60 \pm 0.87 \mathrm{SD}$; see Table 1).

\section{Blood sample analysis}

Serum NGAL analysis revealed significantly increased levels among OCD patients relative to healthy participants (OCD, $n=20$, median (IQR) 91.130 (96.46); CONT, $n=19$, median (IQR) 60.300 (19.27), with female patients presenting significantly higher levels than female controls $(U=18.00 ; p<.05)$. No differences between male and female participants were apparent within each group (see Table 2 for further information). No correlation was found between NGAL levels and severity of symptoms among OCD patients (total YBOCS score $\mathrm{r}_{\mathrm{s}}=.255(p=.278)$; compulsions $\mathrm{r}_{\mathrm{s}}=.245$ $(p=.299)$; obsessions $\left.\mathrm{r}_{\mathrm{s}}=.229(p=.332)\right)$.

\section{Discussion}

Until today, the existing work focusing on the role of lipocalin in psychiatric disorders include studies on depression, especially late-life depression, and Alzheimer's disease. However, and although inflammation has been proposed to be part of the pathophysiological mechanisms behind OCD, with many studies assessing levels of inflammatory markers in OCD patients, no studies to date have included measurements of NGAL levels. In this study, we proposed to assess NGAL levels among OCD patients. So far, this is the first study to do so and to report significant differences between OCD patients and healthy participants, specifically an elevation of the levels of NGAL among patients. We hypothesized NGAL levels would correlate with severity of disease, as it has been observed in studies on depression [23, 40]. However, our data revealed no such association. We have also observed a significant difference in NGAL levels between female patients and controls, but no differences between genders within groups. There are reports of gender differences in inflammatory markers, although not including NGAL, in patients with depressive symptoms and anxiety, which authors hypothesize to be related with different behavioral and inflammatory pathways and possibly with a difference in disease vulnerability [21]. However, our results do not show a difference between genders but a difference among women, and there are no previous reports on gender specific differences in NGAL levels among psychiatric patients. We can only speculate on the variety of factors that might be contributing for our result, for instance differences in disease vulnerability, lifestyle or exposure to environmental factors such as stress. Thus, further studies with 
Table 1 Sample characterization. Values are presented as mean \pm SD for normally distributed variables

\begin{tabular}{|c|c|c|c|c|}
\hline & & OCD group $(N=20)$ & CONT group $(N=19)$ & Statistics \\
\hline$\overline{\mathrm{Age}^{\mathrm{a}}}$ & & $30.50(7.5)$ & $28.00(5)$ & $U=178.0 ; p=.746$ \\
\hline \multirow[t]{2}{*}{ Sex } & Female & $45.0 \%$ & $52.6 \%$ & $X^{2}(1, N=40)=0.227 ; p>.05$ \\
\hline & Male & $55.0 \%$ & $47.4 \%$ & \\
\hline Y-BOCS Total & & $26.10 \pm 1.56$ & - & - \\
\hline Y-BOCS Obsessions & & $14.60 \pm 0.87$ & - & - \\
\hline Y-BOCS Compulsions & & $11.50 \pm 0.76$ & _- & _- \\
\hline
\end{tabular}

CONT control, OCD Obsessive-Compulsive Disorder, SD standard deviation, $Y$-BOCS Yale-Brown Obsessive-Compulsive Scale. ${ }^{\mathrm{a}}$ Values are presented as median (IQR) for non-normally distributed variables

larger samples are needed in order to corroborate this result.

Although our findings seem to differ from results from animal studies, where it is the absence of lipocalin, not the elevation, that is behind the abnormal phenotype and neurochemical alterations $[32,35]$, it is possible that the influence of NGAL follows a U-shaped curve [41], with both the absence and the elevation of levels explaining the behavioral and neurochemical changes. This dual effect has been proposed regarding inflammatory cytokines and their impact on sleep, but this model is yet to be proven [42]. However, studies assessing NGAL function have provided interesting results supporting this notion, where both lipocalin deficiency and overexpression are associated with behavioral impairments. Thus, while a number of animal and human studies have found elevated levels of lipocalin in disease states such as depression [22, 40], schizophrenia [43], multiple sclerosis [20], Parkinson's [31] and Alzheimer's disease [29, 30], suggesting lipocalin overexpression might play an important role in mediating the observed deficits, other authors have reported findings such as increased anxiety- and depressive-like behavior and cognitive impairments displayed by LCN2-null mice [32, $33,35,44]$. On the contrary, a recent study by Mike and colleagues assessing the role of LCN2 on the neurobehavioral manifestations of a mouse lupus model found LCN2 deficiency in Sle1,3 mice attenuated the depressive-like phenotype as well as memory impairments that are characteristic of the model. Most notably, LCN2-KO animals did not exhibit impairments in

Table 2 NGAL levels among participants according to gender. Values are presented as median (IQR) for non-normally distributed variables

\begin{tabular}{llllll}
\hline Gender & $\mathbf{N}$ & OCD group & $\mathbf{N}$ & CONT group & Statistics \\
\hline Male & 11 & $75.430(50.08)$ & 9 & $60.92 \pm 20.36^{\mathrm{a}}$ & $U=30.00 ; p>.05$ \\
Female & 9 & $142.19 \pm 70.39^{\mathrm{a}}$ & 10 & $61.75(31.53)$ & $U=18.00 ; p<.05$ \\
Total & 21 & $91.130(96.46)$ & 19 & $60.300(19.27)$ & $\underline{U}=99.00 ; p=.01$ \\
\hline
\end{tabular}

$O C D$ Obsessive-Compulsive Disorder, IQR interquartile range, CONT control. avalues are presented as mean \pm SD for normally distributed variables exploratory behavior or motor coordination nor showed deficits in the sucrose-preference, object recognition and object placement tests compared to control animals [45], which is in line with other reports where both LCN2 deficient and wild type mice exhibited a similar behavioral phenotype in response to liposaccharide injection [46]. Thus, it seems plausible to admit lipocalin may play different roles according to the physiology or pathological conditions $[47,48]$ and further studies are needed to better understand its role in mediating disease states.

Most importantly, since lipocalin constitutes a marker of inflammation not necessarily associated with infection [48], our results highlight the importance of describing the inflammatory profile of OCD patients, given this might be of relevance for the understanding of the disorder in patients other than those evidencing OCS in the context of prior history of infection. Previous studies have demonstrated how other immune abnormalities might be related to glutamatergic dysfunction within the CSTC circuit, the main pathway associated with OCD development [16, 49]. Additionally, animal and human studies have shown lipocalin to be expressed in a variety of brain regions, including the striatum, thalamus and prefrontal cortex $[31,50]$, regions that have been implicated in OCD pathophysiology. The link between immune dysregulation and OCD is yet to be unraveled, although theories suggest glutamate might play a strategic role given its presence within the CSTC circuit and its involvement in regulating $\mathrm{T}$ cell function [51, 52]. Given the hypothetical nature of such theory, further work is needed in order to better understand the relative contribution of immune dysregulation in OCD pathophysiology, with larger samples, detailed clinical characterization of participants, control for medication effect and rigorous selection criteria as key points to have into account in future studies.

Naturally, our study has some limitations. The use of a small number of patients limits the generalization of results. Additionally, whether the observed increase in NGAL levels is a cause or a consequence of the disorder remains to be defined and should warrant further investigation. Future studies on this subject would also 
benefit of a more detailed assessment of the inflammation status of subjects, with inclusion of other markers of inflammation and the determination of antistreptolysin titers. Thus, it would be interesting to proceed with studies on this subject not only by including larger, well characterized cohorts, but also through the use of animal models, which allow for the dissection of biological mechanisms otherwise impossible to study in humans.

In conclusion, we herein report differences in NGAL levels among OCD patients compared to healthy controls. We offer additional evidence to the immune dysregulation hypothesis of OCD by reporting elevated levels of NGAL among OCD patients versus healthy controls, with higher differences being found among women.

However, while our results warrant further replication, more research is needed in order to clarify the role of NGAL in this neuropsychiatric disorder.

\section{Abbreviations \\ CNS: Central nervous system; CSTC circuit: Cortico-striatal-thalamo-cortical circuit; HPA axis: Hypothalamic-pituitary-adrenal axis; LCN2: Lipocalin-2; MS: Multiple sclerosis; NGAL: Neutrophil gelatinase-associated lipocalin; OCD: Obsessive-compulsive disorder; OCS: Obsessive-compulsive symptoms; Y-BOCS: Yale-Brown Obsessive-Compulsive Scale}

\section{Acknowledgments}

The authors thank all the patients enrolled in this study.

\section{Authors' contributions}

PM recruited and assessed OCD patients. IMP and FM collected and performed blood sample analysis. CRL analyzed the data and wrote the first draft of the manuscript. All authors interpreted the data, read and approved the final manuscript.

\section{Funding}

This work was performed in the Life and Health Sciences Research Institute (ICVS), Minho University. This work has been funded by National funds, through the Foundation for Science and Technology (FCT) - project UIDB/ 50026/2020 and UIDP/50026/2020; and by the projects NORTE-01-0145-FEDE R-000013 and NORTE-01-0145-FEDER-000023, supported by Norte Portugal Regional Operational Programme (NORTE 2020), under the PORTUGAL 2020 Partnership Agreement, through the European Regional Development Fund (ERDF). Catarina Raposo-Lima was supported by fellowship grant from FCT Foundation for Science and Technology fellowship grant to Catarina RaposoLima (reference SFRH/BD/122959/2016).

\section{Availability of data and materials}

The datasets used and/or analyzed during the current study are available from the corresponding author on reasonable request.

\section{Declarations}

\section{Ethics approval and consent to participate}

This study was performed in accordance with the Declaration of Helsinki and was approved by Ethics Subcommittee for the Life and Health Sciences of University of Minho, Portugal, and by the Ethics Committee of the Hospital of Braga, Portugal. All subjects were provided with written informed consent following description of the study goals and procedures.

\section{Consent for publication}

Not applicable.

\section{Competing interests}

P Morgado has received in the past 3 years grants, CME-related honoraria, or consulting fees from Angelini, AstraZeneca, Bial Foundation, Biogen, DGS-
Portugal, FCT, Janssen-Cilag, Gulbenkian Foundation, Lundbeck, Springer Healthcare, Tecnimede and 2CA-Braga. C Raposo-Lima, IM Pereira and F Marques declare no conflict of interests.

\section{Author details}

'Life and Health Sciences Research Institute (ICVS), School of Medicine, University of Minho, 4710-057 Braga, Portugal. ${ }^{2}$ ICVS-3Bs PT Government Associate Laboratory, Braga, Guimarães, Portugal. ${ }^{3}$ Clinical Academic Center-Braga (2CA), Hospital de Braga, Braga, Portugal.

Received: 5 February 2021 Accepted: 30 March 2021

Published online: 26 May 2021

\section{References}

1. American Psychiatric Association. Diagnostic and statistical manual of mental disorders, 5th edition (DSM-5). Arlington, VA: American Psychiatric Association; 2013. https://doi.org/10.1176/appi.books.9780890425596.

2. Raposo-Lima C, Morgado P. The role of environmental factors in the pathogenesis of obsessive-compulsive and related disorders. In: Fontenelle LF, Yücel M, editors. A Transdiagnostic approach to obsessions, compulsions and related phenomena. Cambridge: Cambridge University Press; 2019. p. 39-50. https://doi.org/10.1017/9781108164313.006.

3. Swedo SE, Leonard HL, Garvey M, Mittleman B, Allen AJ, Perlmutter S, et al. Pediatric autoimmune neuropsychiatric disorders associated with streptococcal infections: clinical description of the first 50 cases. Am J Psychiatry. 1998;155(2):264-71. https://doi.org/10.1176/ajp.155.2.264.

4. Cutforth T, DeMille MM, Agalliu I, Agalliu D. CNS autoimmune disease after streptococcus pyogenes infections: animal models, cellular mechanisms and genetic factors. Future Neurol. 2016;11(1):63-76. https://doi.org/10.2217/fnl.16.4.

5. Pearlman DM, Vora HS, Marquis BG, Najjar S, Dudley LA. Anti-basal ganglia antibodies in primary obsessive-compulsive disorder: systematic review and meta-analysis. Br J Psychiatry. 2014;205(1):8-16. https://doi.org/10.1192/bjp. bp.113.137018.

6. Snider LA, Swedo SE. Post-streptococcal autoimmune disorders of the central nervous system. Curr Opin Neurol. 2003;16(3):359-65. https://doi. org/10.1097/01.wCo.0000073938.19076.31.

7. Gerentes M, Pelissolo A, Rajagopal K, Tamouza R, Hamdani N. Obsessivecompulsive disorder: autoimmunity and Neuroinflammation. Curr Psychiatry Rep. 2019;21(8):78. https://doi.org/10.1007/s11920-019-1062-8.

8. Mora S, Martín-González E, Flores P, Moreno M. Neuropsychiatric consequences of childhood group a streptococcal infection: a systematic review of preclinical models. Brain Behav Immun. 2020;86:53-62. https://doi. org/10.1016/j.bbi.2019.02.027.

9. Martino D, Johnson I, Leckman JF. What does immunology have to do with Normal brain development and the pathophysiology underlying Tourette syndrome and related neuropsychiatric disorders? Front Neurol. 2020;11: 1101

10. Mitchell $\mathrm{RH}$, Goldstein BI. Inflammation in children and adolescents with neuropsychiatric disorders: a systematic review. J Am Acad Child Adolesc Psychiatry. 2014;53(3):274-96. https://doi.org/10.1016/j.jaac.2013.11.013.

11. Rao NP, Venkatasubramanian G, Ravi V, Kalmady S, Cherian A, YC JR. Plasma cytokine abnormalities in drug-naïve, comorbidity-free obsessivecompulsive disorder. Psychiatry Res. 2015;229(3):949-52. https://doi.org/10.1 016/j.psychres.2015.07.009.

12. Rodríguez N, Morer A, González-Navarro EA, Serra-Pages C, Boloc D, Torres $T$, et al. Inflammatory dysregulation of monocytes in pediatric patients with obsessive-compulsive disorder. J Neuroinflammation. 2017;14(1):261. https://doi.org/10.1186/s12974-017-1042-z.

13. Attwells S, Setiawan E, Wilson AA, Rusjan PM, Mizrahi R, Miler L, et al. Inflammation in the neurocircuitry of obsessive-compulsive disorder. JAMA Psychiatry. 2017;74(8):833-40. https://doi.org/10.1001/jamapsychia try.2017.1567.

14. Cosco TD, Pillinger T, Emam H, Solmi M, Budhdeo S, Prina AM, et al. Immune aberrations in obsessive-compulsive disorder: a systematic review and meta-analysis. Mol Neurobiol. 2019:56(7):4751-9. https://doi.org/10.1 007/s12035-018-1409-X.

15. Renna ME, O'Toole MS, Spaeth PE, Lekander M, Mennin DS. The association between anxiety, traumatic stress, and obsessive-compulsive disorders and chronic inflammation: a systematic review and meta-analysis. Depress Anxiety. 2018;35(11):1081-94. https://doi.org/10.1002/da.22790. 
16. Dietrich D, Zhang Y, Bode L, Münte T, Hauser U, Schmorl P, et al. Brain potential amplitude varies as a function of Borna disease virus-specific immune complexes in obsessive-compulsive disorder. Mol Psychiatry. 2005; 10(6):519-20. https://doi.org/10.1038/sj.mp.4001645.

17. Liu Q, Nilsen-Hamilton M. Identification of a new acute phase protein. J Biol Chem. 1995;270(38):22565-70. https://doi.org/10.1074/jbc.270.38.22565.

18. Bao G, Clifton M, Hoette TM, Mori K, Deng S-X, Qiu A, et al. Iron traffics in circulation bound to a siderocalin (Ngal)-catechol complex. Nat Chem Biol. 2010;6(8):602-9. https://doi.org/10.1038/nchembio.402.

19. Ferreira AC, Da Mesquita S, Sousa JC, Correia-Neves M, Sousa N, Palha JA, et al. From the periphery to the brain: Lipocalin-2, a friend or foe? Prog Neurobiol. 2015;131:120-36. https://doi.org/10.1016/j.pneurobio.2015.06.005.

20. Marques F, Mesquita SD, Sousa JC, Coppola G, Gao F, Geschwind DH, et al. Lipocalin 2 is present in the EAE brain and is modulated by natalizumab. Front Cell Neurosci. 2012;6:33.

21. Mommersteeg PM, Naudé PJ, Bagijn W, Widdershoven J, Westerhuis BW, Schoemaker RG. Gender differences in associations of depressive symptoms and anxiety with inflammatory markers in patients with non-obstructive coronary artery disease. J Psychosom Res. 2019;125:109779. https://doi.org/1 0.1016/j.jpsychores.2019.109779.

22. Naudé P, Eisel U, Comijs H, Groenewold N, De Deyn P, Bosker F, et al. Neutrophil gelatinase-associated lipocalin: a novel inflammatory marker associated with late-life depression. J Psychosom Res. 2013;75(5):444-50. https://doi.org/10.1016/j.jpsychores.2013.08.023.

23. Naudé PJ, Mommersteeg PM, Zijlstra WP, Gouweleeuw L, Kupper N, Eisel $U L$, et al. Neutrophil gelatinase-associated lipocalin and depression in patients with chronic heart failure. Brain Behav Immun. 2014;38:59-65. https://doi.org/10.1016/j.bbi.2013.12.023.

24. Veltman E, Lamers F, Comijs H, Stek M, Van der Mast R, Rhebergen D. Inflammatory markers and cortisol parameters across depressive subtypes in an older cohort. J Affect Disord. 2018;234:54-8. https://doi.org/10.1016/j.ja d.2018.02.080.

25. Marques F, Rodrigues A-J, Sousa JC, Coppola G, Geschwind DH, Sousa N, et al. Lipocalin 2 is a choroid plexus acute-phase protein. J Cereb Blood Flow Metab. 2008;28(3):450-5. https://doi.org/10.1038/sj.jcbfm.9600557.

26. MacManus JP, Graber T, Luebbert C, Preston E, Rasquinha I, Smith B, et al. Translation-state analysis of gene expression in mouse brain after focal ischemia. J Cereb Blood Flow Metab. 2004;24(6):657-67. https://doi.org/10.1 097/01.WCB.0000123141.67811.91

27. De Biase A, Knoblach SM, Di Giovanni S, Fan C, Molon A, Hoffman EP, et al. Gene expression profiling of experimental traumatic spinal cord injury as a function of distance from impact site and injury severity. Physiol Genomics. 2005;22(3):368-81. https://doi.org/10.1152/physiolgenomics.00081.2005.

28. Poh K-W, Yeo J-F, Stohler CS, Ong W-Y. Comprehensive gene expression profiling in the prefrontal cortex links immune activation and neutrophil infiltration to antinociception. J Neurosci. 2012;32(1):35-45. https://doi.org/1 0.1523/JNEUROSCI.2389-11.2012.

29. Mesquita S, Ferreira A, Falcao A, Sousa J, Oliveira TG, Correia-Neves M, et al. Lipocalin 2 modulates the cellular response to amyloid beta. Cell Death Differ. 2014;21(10):1588-99. https://doi.org/10.1038/cdd.2014.68.

30. Naudé PJ, Nyakas C, Eiden LE, Ait-Ali D, Van Der Heide R, Engelborghs S, et al. Lipocalin 2: novel component of proinflammatory signaling in Alzheimer's disease. FASEB J. 2012;26(7):2811-23. https://doi.org/10.1096/ fj.11-202457.

31. Kim B-W, Jeong KH, Kim J-H, Jin M, Kim J-H, Lee M-G, et al. Pathogenic upregulation of glial lipocalin-2 in the parkinsonian dopaminergic system. Neurosci. 2016;36(20):5608-22. https://doi.org/10.1523/JNEUROSCl.4261-15.2016.

32. Ferreira AC, Pinto V, Mesquita SD, Novais A, Sousa JC, Correia-Neves M, et al. Lipocalin-2 is involved in emotional behaviors and cognitive function. Front Cell Neurosci. 2013;7:122.

33. Mucha M, Skrzypiec AE, Schiavon E, Attwood BK, Kucerova E, Pawlak R. Lipocalin-2 controls neuronal excitability and anxiety by regulating dendritic spine formation and maturation. Proc Natl Acad Sci U S A. 2011;108(45): 18436-41. https://doi.org/10.1073/pnas.1107936108.

34. Skrzypiec AE, Shah RS, Schiavon E, Baker E, Skene N, Pawlak R, et al. Stressinduced lipocalin-2 controls dendritic spine formation and neuronal activity in the amygdala. PLoS One. 2013;8(4):e61046.

35. Ferreira A, Santos T, Sampaio-Marques B, Novais A, Mesquita S, Ludovico P, et al. Lipocalin-2 regulates adult neurogenesis and contextual discriminative behaviours. Mol Psychiatry. 2018:23(4):1031-9. https://doi.org/10.1038/mp.2 017.95 .
36. Rodrigues A-J, Leão P, Carvalho M, Almeida OF, Sousa N. Potential programming of dopaminergic circuits by early life stress. Psychopharmacology. 2011;214(1):107-20. https://doi.org/10.1007/s00213-01 0-2085-3.

37. Castro-Rodrigues P, Camacho M, Almeida S, Marinho M, Soares C, BarahonaCorrêa JB, et al. Criterion validity of the Yale-Brown obsessive-compulsive scale second edition for diagnosis of obsessive-compulsive disorder in adults. Front Psychiatry. 2018;9:431. https://doi.org/10.3389/fpsyt.2018.00431

38. Goodman WK, Price LH, Rasmussen SA, Mazure C, Fleischmann RL, Hill CL, et al. The Yale-Brown obsessive compulsive scale: I. development, use, and reliability. Arch Gen Psychiatry. 1989;46(11):1006-11. https://doi.org/10.1 001/archpsyc.1989.01810110048007.

39. Corp I. SPSS statistics for Macintosh, version 27.0. IBM Corp: Armonk, NY; 2020

40. Naudé PJ, Mommersteeg PM, Gouweleeuw L, Eisel UL, Denollet J, Westerhuis $L W$, et al. NGAL and other markers of inflammation as competitive or complementary markers for depressive symptom dimensions in heart failure. World J Biol Psychiatry. 2015;16(7):536-41. https://doi.org/10.3109/15622975.2015.1062550.

41. Gouweleeuw L, Naudé P, Rots M, DeJongste M, Eisel U, Schoemaker R. The role of neutrophil gelatinase associated lipocalin (NGAL) as biological constituent linking depression and cardiovascular disease. Brain Behav Immun. 2015;46:23-32. https://doi.org/10.1016/j.bbi.2014.12.026.

42. Pollmächer T, Haack M, Schuld A, Reichenberg A, Yirmiya R. Low levels of circulating inflammatory cytokines - do they affect human brain functions? Brain Behav Immun. 2002;16(5):525-32. https://doi.org/10.1016/S0889-1591 (02)00004-1.

43. Wei L, Du Y, Wu W, Fu X, Xia Q. Elevation of plasma neutrophil gelatinaseassociated lipocalin (NGAL) levels in schizophrenia patients. J Affect Disord. 2018;226:307-12. https://doi.org/10.1016/j.jad.2017.10.002.

44. Kang SS, Ren Y, Liu C-C, Kurti A, Baker KE, Bu G, et al. Lipocalin-2 protects the brain during inflammatory conditions. Mol Psychiatry. 2018;23(2):344-50. https://doi.org/10.1038/mp.2016.243.

45. Mike EV, Makinde HM, Gulinello M, Vanarsa K, Herlitz L, Gadhvi G, et al. Lipocalin-2 is a pathogenic determinant and biomarker of neuropsychiatric lupus. J Autoimmun. 2019;96:59-73. https://doi.org/10.1016/j.jaut.2018.08. 005.

46. Vichaya EG, Gross PS, Estrada DJ, Cole SW, Grossberg AJ, Evans SE, et al. Lipocalin-2 is dispensable in inflammation-induced sickness and depressionlike behavior. Psychopharmacology. 2019;236(10):2975-82. https://doi.org/1 0.1007/s00213-019-05190-7.

47. Bhusal A, Rahman MH, Lee W-H, Bae YC, Lee I-K, Suk K. Paradoxical role of lipocalin-2 in metabolic disorders and neurological complications. Biochem Pharmacol. 2019;113626.

48. Pinyopornpanish K, Chattipakorn N, Chattipakorn SC. Lipocalin-2: its perspectives in brain pathology and possible roles in cognition. J Neuroendocrinol. 2019;31(10):e12779. https://doi.org/10.1111/jne.12779.

49. Bhattacharyya S, Khanna S, Chakrabarty K, Mahadevan A, Christopher R, Shankar S. Anti-brain autoantibodies and altered excitatory neurotransmitters in obsessive-compulsive disorder. Neuropsychopharmacology. 2009;34(12):2489-96. https://doi.org/10.1038/ npp.2009.77.

50. Dekens DW, Naudé PJ, Engelborghs S, Vermeiren Y, Van Dam D, Oude Voshaar RC, et al. Neutrophil gelatinase-associated lipocalin and its receptors in Alzheimer's disease (AD) brain regions: differential findings in AD with and without depression. J Alzheimers Dis. 2017;55(2):763-76. https://doi.org/10.3233/JAD-160330.

51. Rotge J, Aouizerate B, Tignol J, Bioulac B, Burbaud P, Guehl D. The glutamate-based genetic immune hypothesis in obsessive-compulsive disorder. An integrative approach from genes to symptoms. Neuroscience. 2010;165(2):408-17. https://doi.org/10.1016/j.neuroscience.2009.10.043.

52. Wu K, Hanna GL, Rosenberg DR, Arnold PD. The role of glutamate signaling in the pathogenesis and treatment of obsessive-compulsive disorder. Pharmacol Biochem Behav. 2012;100(4):726-35. https://doi.org/10.1016/j. pbb.2011.10.007.

\section{Publisher's Note}

Springer Nature remains neutral with regard to jurisdictional claims in published maps and institutional affiliations. 